\title{
Modern high strength steels under high strain-rate regimes
}

\author{
Ezio Cadoni ${ }^{1, *}$, Matteo Dotta $^{1}$, and Daniele Forni ${ }^{1}$ \\ ${ }^{1}$ DynaMat SUPSI Laboratory, University of Applied Sciences and Arts of Southern Switzerland, Via \\ Francesco Catenazzi 23, 6850 Mendrisio, Switzerland.
}

\begin{abstract}
In order to properly design critical infrastructures and buildings in steel (bridges, high-rise building, off-shore, cranes, etc.), certain requirements concerning to mechanical resistance and robustness under exceptional actions have to be carefully fulfilled. An acceptable level of safety must be assured to avoid human loss, environmental pollution and material damage. These structures can be subjected to severe accidental loading such as blast or impact. In this context it is fundamental to adequately know the behaviour of structural steel under high strain rate. Modern high strength steels are quenched and selftempered steels. These steels have several layers with differentiated microstructures (martensitic in the cortical part and ferritic in the core). The behaviour of the single layer at high strain rate regimes have to be accurately studied. The paper collects and discusses the tensile results at high strain rate obtained on samples of homogeneous layers of S690QL and S960QL steels. Finally, the characterisation of the single layers has been used in order to analyse the results obtained in large specimen obtained from slabs $12 \mathrm{~mm}$ thick.
\end{abstract}

\section{Introduction}

In the steel structures design and in particular in special cases (i.e. off-shore, large span bridge, high-rise building), impact and blast have become important issues and enhanced understanding of plastic deformation of material at high rate is required. Currently, the use of High Strength Steel (HSS) and Very High Strength Steel (VHSS) in construction of modern and advanced structure is in sustained expansion. Compared to the traditional grade steels, they have superior mechanical characteristics hence the weight savings and lower life cycle cost (minor costs for construction and maintenance). These steels are manufactured by means of quenching and tempering, this process produces the differentiation of materials from the outer part (martensitic microstructure) to the inner part (bainitic-ferritic microstructures). Consequently, these steels have a differentiated micro-structure and behave as a functional graded material. For this reason it is fundamental to know the mechanical response of the different homogeneous parts at various strain rate regimes. In this paper are collected and compared recent results obtained on two different HSS and VHSS steels (S690QL and S960QL) $[1,2]$ in a wide strain rate interval ranging from $10^{-3} \mathrm{~s}^{-1}$ to $10^{3} \mathrm{~s}^{-1}$. Finally, the characterisation of the single layers has been used in order to analyse the results obtained in large specimens obtained from slabs $12 \mathrm{~mm}$ thick.

\footnotetext{
*e-mail: ezio.cadoni@supsi.ch
} 


\section{Material and specimens}

Outer and inner zone (Figure 1a) specimens were obtained from commercially available S690QL and S960QL plates $40 \mathrm{~mm}$ thick vertically cut (Figure 1b) by means of WEDM (Wire-Electrical Discharge Machining) as described in [2]. The high strain rate tests were performed on a round specimen having a diameter of $3 \mathrm{~mm}$ and gauge length of $5 \mathrm{~mm}$ as shown in Figure 1c. The specimen ends had a M5 fillet for the connection to the testing machines. The inner part shows a bainitic structure, while the outer part presents a martensitic ones. In order to accurately establish which area investigate a hardness measurements scan on the whole section was performed. Figure 2 shows Hardness Vickers(HV30) measurements in a normalised thickness. They highlight difference between the outer and the inner part. As a consequence the samples were extracted in the longitudinal direction from steel plates from two different positions as highlighted in Figure 1.
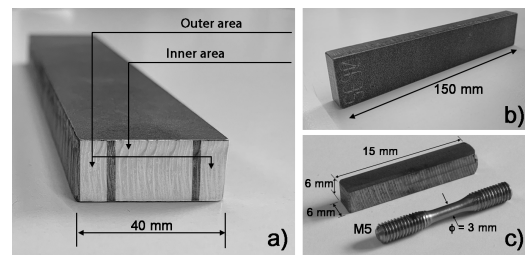

Figure 1. Preparation of the specimens from plate $40 \mathrm{~mm}$ thick.

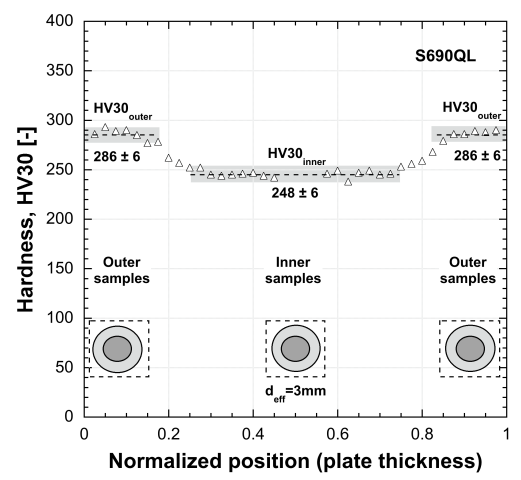

Figure 2. Hardness measurements HV30 within the normalised thickness.

\section{Experimental methodology for high strain-rate regime}

High strain-rate behaviour of the inner and outer specimens of S690QL [1] and S960QL [2] were investigated by means of a Split Hopkinson Tensile Bar (SHTB) [3, 4]. With reference to Figure 3 where the SHTB is shown, the experimental set up is composed by a hydraulic actuator (1), a circular straight high strength steel bar with a diameter of $10 \mathrm{~mm}$ and a length of $9 \mathrm{~m}$ (2) used for the first $6 \mathrm{~m}$ as a pretensioned bar, after the blocking device (3), the remaining $3 \mathrm{~m}$ as input bar (4). The input bar is instrumented with a semiconductor strain gauge station (5) in order to register the incident and reflected pulses acting on sample (6) screwed in the 
input and the output bar (7). The latest is also instrumented with semiconductor strain gauges (8) in order to register the transmitted pulse. The test velocity is ruled by the stress level in the pretensioned bar in which the elastic energy is stored thanks to the hydraulic jack and the blocking device.

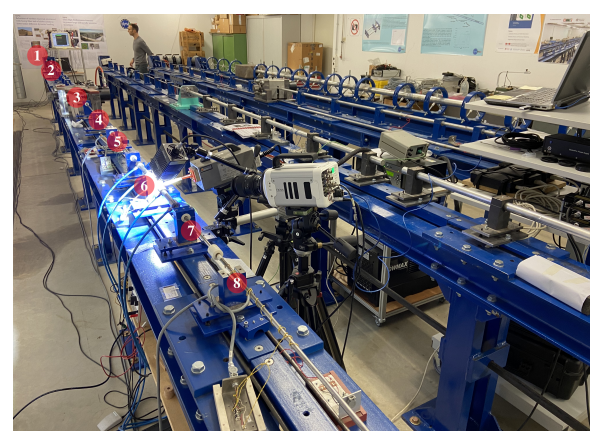

Figure 3. Dynamic testing set-up.

From semiconductor strain gauges the incident $\left(\epsilon_{I}\right)$, reflected $\left(\epsilon_{R}\right)$ and transmitted $\left(\epsilon_{T}\right)$ pulses acting on the cross section of the specimen are obtained. By applying the onedimensional elastic plane stress wave propagation theory the engineering value of stress (1), strain (2) and strain-rate (3) are obtained in function of time:

$$
\begin{gathered}
\sigma_{\text {eng }}(t)=E_{0} \cdot \frac{A_{0}}{A} \cdot \epsilon_{T}(t) \\
\epsilon_{\text {eng }}(t)=-\frac{2 C_{0}}{L} \int_{0}^{t} \epsilon_{R}(t) \\
\dot{\epsilon}_{\text {eng }}(t)=-\frac{2 C_{0}}{L} \cdot \epsilon_{R}(t)
\end{gathered}
$$

Three target high strain-rates were set at $250 \mathrm{~s}^{-1}, 450 \mathrm{~s}^{-1}$ and $950 \mathrm{~s}^{-1}$ obtained by imposing different preloads for outer and inner sample of S690QL and S960QL. During the tests the signals from strain-gauges were acquired by a HBM-Gen2 data acquisition system while the specimens were filmed at high speed by a fast camera Photron S12 at 50kfps.

\section{Results}

Figures 4 a,b depict the engineering stress versus strain curves of the different layers in quasistatic and dynamic regimes, respectively.

As shown in Figure 5, the proof and ultimate tensile strengths increase with increasing strain-rates for both inner and outer part. For S690QL the dynamic yield strength is about 1.21 and 1.11 times the values obtained in quasi-static conditions for inner and outer zone, respectively, while for S960QL the values are 1.12 and 1.09. For S690QL the dynamic ultimate tensile strength is about 1.14 and 1.12 times the values obtained in quasi-static conditions for inner and outer part, respectively. In the case of S960QL the values are 1.08 and 1.09. In Tables 1, 2, 3 and 4 are resumed the most important parameters obtained in $[1,2]$ for S690QL and S960QL inner and outer parts. 

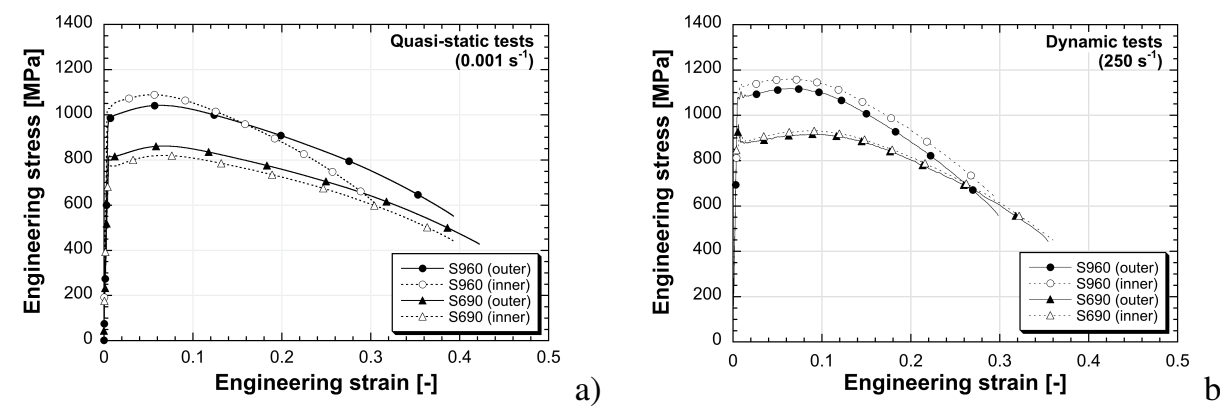

Figure 4. Stress versus strain curves: a) quasi-static tests; b) dynamic tests (250 1/s).
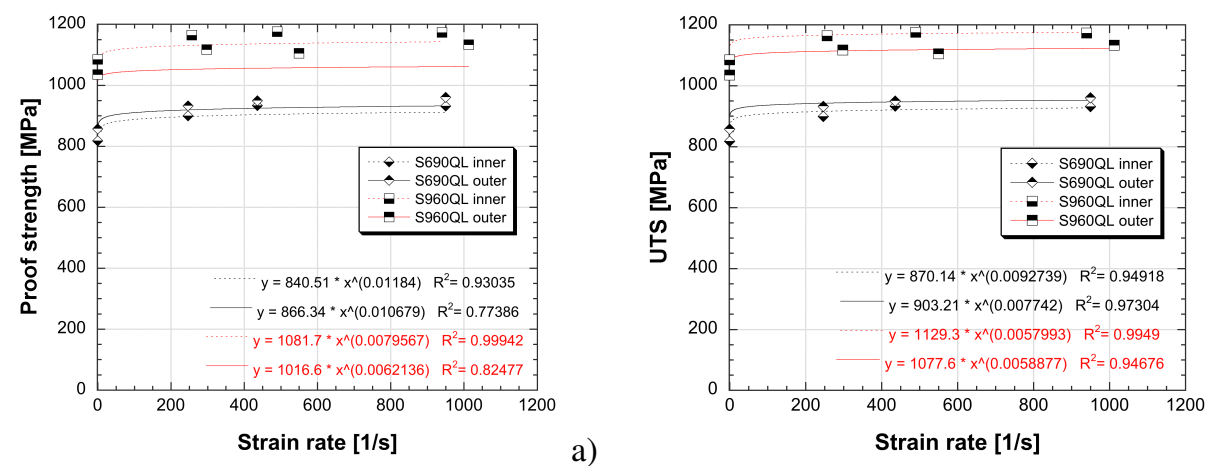

b)

Figure 5. Proof strength (a) and UTS (b) versus strain rate curves.

Table 1. S690QL inner part results at different strain rate [1]

\begin{tabular}{lllll}
\hline Effective strain-rate, $\dot{\epsilon}\left(\mathrm{s}^{-1}\right)$ & 0.001 & $247 \pm 18$ & $436 \pm 29$ & $939 \pm 8$ \\
\hline Reduction of area, $Z(\%)$ & $73 \pm 1$ & $72.9 \pm 0.6$ & $73.0 \pm 1.9$ & $75.0 \pm 0.6$ \\
Proof strength, $f_{p, 0.2 \%}(\mathrm{MPa})$ & $775 \pm 5$ & $878 \pm 18$ & $927 \pm 16$ & $907 \pm 10$ \\
Ult. tensile strength, $f_{u}(\mathrm{MPa})$ & $817 \pm 6$ & $899 \pm 13$ & $933 \pm 6$ & $931 \pm 6$ \\
Uniform strain, $\epsilon_{u}(\%)$ & $4.0 \pm 0.4$ & $8.9 \pm 0.9$ & $8.8 \pm 0.4$ & $9.6 \pm 1.0$ \\
Eng. fract. strength, $f_{f}(\mathrm{MPa})$ & $434 \pm 18$ & $442 \pm 2$ & $464 \pm 11$ & $443 \pm 3$ \\
Eng. fract. strain, $\epsilon_{f}(\%)$ & $20.1 \pm 1.1$ & $37.2 \pm 2.5$ & $35.7 \pm 2.1$ & $39.2 \pm 0.4$ \\
\hline
\end{tabular}

\section{Assessment on large specimens}

In order to assess the global behaviour of specimens (see Figure 6) coming from a $12 \mathrm{~mm}$ thick slab a specific experimental campaign was carried our in the frame of project named Steel Under Severe High Impact (SUSHI) developed in the Framework of access to the Joint Research Centre Physical Research Infrastructures of the European Commission. The tests were carried out by means of the large Hopkinson bar (today named HopLab) installed in the JRC at Ispra (Italy). This special infrastructure is $200 \mathrm{~m}$ long, the loading pulse is generated by means of 32 high strength steel cables connected to the input bar having $72 \mathrm{~mm}$ in diameter and $12 \mathrm{~m}$ in length while the output bar has same diameter but $90 \mathrm{~m}$ in length. With this apparatus a rectangular loading pulse characterized by $1 \mathrm{MN}$ amplitude, $250 \mu$ s rise-time and $40 \mathrm{~ms}$ duration can be generated. Among the different performed experiments the two tests 
Table 2. S690QL outer part results at different strain rate [1]

\begin{tabular}{lllll}
\hline Effective strain-rate, $\dot{\epsilon}\left(\mathrm{s}^{-1}\right)$ & 0.001 & $208 \pm 15$ & $398 \pm 22$ & $849 \pm 6$ \\
\hline Reduction of area, $Z(\%)$ & $72.0 \pm 0.1$ & $74.0 \pm 0.7$ & $73.1 \pm 1.3$ & $71.7 \pm 1.7$ \\
Proof strength, $f_{p, 0.2 \%}(\mathrm{MPa})$ & $808 \pm 4$ & $872 \pm 26$ & $942 \pm 50$ & $960 \pm 9$ \\
Ult. tensile strength, $f_{u}(\mathrm{MPa})$ & $857 \pm 5$ & $932 \pm 5$ & $949 \pm 8$ & $960 \pm 4$ \\
Uniform strain, $\epsilon_{u}(\%)$ & $6.6 \pm 0.3$ & $8.8 \pm 0.4$ & $8.0 \pm 0.3$ & $9.3 \pm 0.7$ \\
Eng. fract. strength, $f_{f}(\mathrm{MPa})$ & $435 \pm 8$ & $456 \pm 11$ & $442 \pm 13$ & $432 \pm 4$ \\
Eng. fract. strain, $\epsilon_{f}(\%)$ & $41.3 \pm 0.8$ & $35.3 \pm 1.1$ & $35.1 \pm 1.5$ & $36.1 \pm 0.2$ \\
\hline
\end{tabular}

Table 3. S960QL inner part results at different strain rate [2]

\begin{tabular}{lllll}
\hline Effective strain rate, $\dot{\epsilon}\left(\mathrm{s}^{-1}\right)$ & 0.001 & $257 \pm 23$ & $490 \pm 34$ & $940 \pm 44$ \\
\hline Reduction of area, $Z(\%)$ & $70.0 \pm 0.4$ & $66.3 \pm 1.2$ & $66.2 \pm 2.0$ & $68.5 \pm 1.0$ \\
Proof strength, $f_{p, 0.2 \%}(\mathrm{MPa})$ & $1024 \pm 2$ & $1129 \pm 10$ & $1136 \pm 11$ & $1144 \pm 3$ \\
Ult. tensile strength, $f_{u}(\mathrm{MPa})$ & $1085 \pm 3$ & $1164 \pm 9$ & $1175 \pm 8$ & $1173 \pm 5$ \\
Uniform strain, $\epsilon_{u}(\%)$ & $6.05 \pm 0.6$ & $5.99 \pm 0.7$ & $6.88 \pm 0.7$ & $6.57 \pm 0.03$ \\
Eng. fract. strength, $f_{f}(\mathrm{MPa})$ & $618 \pm 8$ & $647 \pm 21$ & $646 \pm 9$ & $639 \pm 10$ \\
Eng. fract. strain, $\epsilon_{f}(\%)$ & $31.1 \pm 1.1$ & $29.4 \pm 3.0$ & $30.4 \pm 2.4$ & $27.9 \pm 1.7$ \\
\hline
\end{tabular}

Table 4. S960QL outer part results at different strain rate [2]

\begin{tabular}{lllll}
\hline Effective strain rate, $\dot{\epsilon}\left(\mathrm{s}^{-1}\right)$ & 0.001 & $298 \pm 5$ & $550 \pm 35$ & $1013 \pm 22$ \\
\hline Reduction of area, $Z(\%)$ & $71.9 \pm 0.4$ & $69.8 \pm 0.9$ & $68.5 \pm 2.0$ & $67.1 \pm 5.7$ \\
Proof strength, $f_{p, 0.2 \%}(\mathrm{MPa})$ & $973 \pm 9$ & $1076 \pm 4$ & $1033 \pm 61$ & $1064 \pm 3$ \\
Ult. tensile strength, $f_{u}(\mathrm{MPa})$ & $1035 \pm 6$ & $1117 \pm 4$ & $1105 \pm 23$ & $1133 \pm 22$ \\
Uniform strain, $\epsilon_{u}(\%)$ & $6.3 \pm 0.1$ & $6.5 \pm 0.1$ & $8.0 \pm 0.1$ & $8.1 \pm 0.0$ \\
Eng. fract. strength, $f_{f}(\mathrm{MPa})$ & $557 \pm 25$ & $555 \pm 4$ & $583 \pm 40$ & $611 \pm 120$ \\
Eng. fract. strain, $\epsilon_{f}(\%)$ & $39.6 \pm 0.3$ & $31.1 \pm 1.1$ & $34.1 \pm 2.8$ & $33.0 \pm 1.7$ \\
\hline
\end{tabular}

with highest preloading conditions have been selected, where the strain rate was in the range $200-250 \mathrm{~s}^{-1}$.

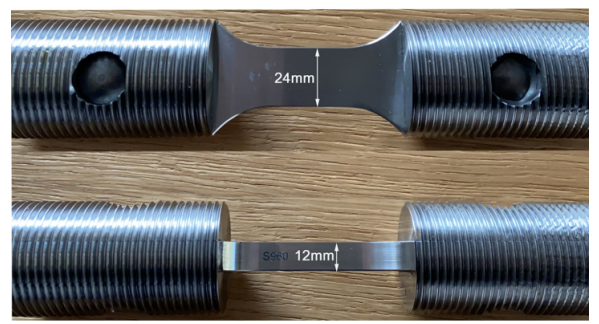

Figure 6. Large specimen for dynamic testing.

Many of two/multi-phased graded materials can be homogenised as a function of the distribution of volume fraction of different phase constituents. Finite element analysis of two/multi-phased graded materials can results problematic. Hence, it is beneficial to use homogenisation schemes starting from the properties of each layer, thickness and volume fractions of phase constituents. Among the most used homogenisation model, that proposed by Tamura-Tomota-Ozawa (TTO model) [5] is based on the assumption that, the magnitude 
Table 5. Comparison between homogenization model and large specimen tests.

\begin{tabular}{llllll}
\hline Material & $\begin{array}{l}\text { Strain rate } \\
\left(\mathrm{s}^{-1}\right)\end{array}$ & $\begin{array}{l}\sigma_{\text {inner }} \\
(\mathrm{MPa})\end{array}$ & $\begin{array}{l}\sigma_{\text {outer }} \\
(\mathrm{MPa})\end{array}$ & $\begin{array}{l}\sigma_{\text {model }} \\
(\mathrm{MPa})\end{array}$ & $\begin{array}{l}\sigma_{\text {exp }} \\
(\mathrm{MPa})\end{array}$ \\
\hline S690QL & $3 \cdot 10^{-5}$ & 718 & 851 & 765 & 790 \\
S690QL & 250 & 899 & 932 & 910 & 887 \\
S960QL & $3 \cdot 10^{-5}$ & 1042 & 1039 & 1041 & 1069 \\
S960QL & 250 & 1164 & 1117 & 1147 & 1145 \\
\hline
\end{tabular}

of stress on every area of a two phased material is dependent on stress of phase constituents undergone with their volume fractions as given in Eq. 4:

$$
\sigma_{\text {eqv }}=\sigma_{\text {inner }} \cdot V_{\text {inner }}+\sigma_{\text {outer }} \cdot V_{\text {outer }}
$$

where: $\sigma_{\text {eqv }}$ is the stress of the homogenised material; $\sigma_{\text {inner }}$ and $\sigma_{\text {outer }}$ are the strength of the core and peripheral layer, respectively; $V_{\text {inner }}$ and $V_{\text {outer }}$ are the volume determined on the basis of the micro-hardness measurements. From Figure 2 can be easyly obtaine the vollowing values: $V_{\text {inner }}=0.65$ and $V_{\text {outer }}=0.35$.

In Table 5 are reported the comparison between homogenisation model and experiments on large specimens. The comparison has been made on the basis of the results obtained in [6] for quasi-static regime and in [1] and [2] for the dynamic response of S690QL and S960QL inner and outer layers, respectively. It can be noted how the TTO model in quasi-static regime overestimates the strength of $3.27 \%$ and $2.69 \%$ while in dynamic regime underestimates of $2.53 \%$ and $0.17 \%$ for S690QL and S960QL, respectively. One of the further steps will be compared the results with others and more sophisticated model such as exponential law model, Voigt model, sigmoid law model, etc.

\section{Conclusions}

In this paper, the high strain rate results on a very-high strength steel, namely S960QL, and a high strength steel, namely S690QL, have been presented. These results obtained on inner and outer part have been used to verify one homogenisation model thanks to the tests carried out on large specimens.

Acknowledgements: The experimental data at high strain rate on large specimens used in this research were generated through access to the HopLab (Hopkinson Bar facility) under the Framework of access to the Joint Research Centre Physical Research Infrastructures of the European Commission (SUSHI project, Research Infrastructure Access Agreement Nr. 35083-2). Any opinions, findings and conclusions or recommendations expressed in this paper are those of the authors and do not necessarily reflect those of the European Commission.

\section{References}

[1] Cadoni E., Forni D. Journal of Constructional Steel Research 175, 106348 (2020)

[2] Cadoni E., Forni D. Fire Safety Journal, 102869 (2019)

[3] Cadoni E., Dotta M., Forni D., Tesio N., Albertini C. Mat. Des.,49 657 - 666 (2013)

[4] Forni D., Chiaia B., Cadoni E. Engineering Structures 119 164-173 (2016)

[5] Tamura I., Tomato Y., Ozawa H. In: Proc. 3rd conf. on strength of metals and alloys, 1. Cambridge: Institute of Metals, 611-615 (1973)

[6] Neuenschwander M., Scandella C., Knobloch M., Fontana M. Mat. Des. 136 81-102 (2017) 